\title{
The current status of bariatric surgery
}

\author{
Stephen Pollard
}

See linked paper on p82

\section{Correspondence to}

Mr S Pollard, St James University Hospital, Beckett Street, Leeds LS9 7TF, UK; stephen.pollard@leedsth.nhs.uk

Accepted 4 January 2011 Published Online First

7 February 2011
As the incidence of obesity continues to rise, increasing numbers of patients are undergoing bariatric surgery to address morbid obesity and weight related health issues. Bariatric procedures aim to reduce dietary intake and/ or interfere with food absorption and are now in widespread use but with a huge capacity shortfall leading to a degree of rationing of the resource. Such treatment should be regarded as palliative in that it does not cure the underlying disorder, and guidelines have been produced to define which patients should be considered for this type of surgery, which must be undertaken in a multidisciplinary setting. Long term results show this to be a cost-effective intervention with a durable positive impact on cardiac risk factors and in particular type 2 diabetes and obstructive sleep apnoea, together with a reduction in all cause mortality and malignancy and an improvement in quality of life. Systematic data collection has now started in the UK and will assist in defining the best application of the resource.

\section{Introduction and definitions}

In the UK, the Registrar General's figures suggest that annually $30000-50000$ people die prematurely from the complications of obesity. This is chiefly through obesity related comorbid conditions which lead to an increased risk of coronary heart disease and an increased risk of certain cancers, such as uterus and colon, and it is estimated that morbid obesity shortens a patient's life expectancy by an average of about 10 years. ${ }^{12}$

The term bariatric is derived from the Greek words baros (pleural bari), meaning weight, burden, load or heaviness, and iatreia meaning healing or care. Bariatric surgery is a collective term for procedures which establish weight loss through surgical interventions. All such operations are tools to aid weight loss and must be combined with regular exercise and improved dietary choices to give the best results.
Obesity is defined by the body mass index (BMI), the result of dividing the weight in kilograms by the height in metres squared. For health, the BMI should be maintained in the range of 18.5-25. If the BMI exceeds 25, a person is overweight, if it exceeds 30 they are defined as obese and if it exceeds 40 (or 35 with obesity related medical problems), a person is morbidly obese, and superobese if the BMI is over $50 .^{3}$ Although most clinicians and indeed some of the general public are familiar with this formula, it has been criticised as being unscientific and biases patients of shorter stature to a disproportionately higher BMI for any particular value of excess weight and there is evidence that waist:hip ratio and absolute waist circumference correlate better with cardiovascular and type II diabetes risk, and mortality. ${ }^{4-6}$

The BMI, as derived from the above formula, is more correctly a unit of pressure, and was invented by the 19th century Belgian mathematician Adolphe Quetelet to quantify the pressure applied to the seat of office chairs, the pressure being applied to the seat, equal to the weight divided by the contact area, being proportional to a person's BMI. Mathematically, the figure derived from height/weight ${ }^{3}$ would be more consistent a measure of obesity irrespective of patient habitus but is unlikely to come into widespread use. ${ }^{7}$

\section{Patient selection}

The National Institute for Clinical Excellence (NICE, now the National Institute for Health and Clinical Excellence) published guidelines for obesity surgery in December 2002 which were updated in 2006 and are generally accepted as the guidelines to which units undertaking obesity surgery should adhere. ${ }^{8}$ NICE support surgery if a patient is morbidly obese-that is, if BMI exceeds 40 , or 35 with two or 
more weight related comorbidities. Table 1 lists the weight related comorbidities. The established presence of gynaecological malignancy or gallstones, which cannot be reversed by weight loss, should not be included for the purposes of fulfilling the NICE criteria although weight loss will reduce the risks of these conditions developing. ${ }^{9}$

NICE did not recommend or compare individual procedures. NICE recommended that prior to surgery, patients should be considered for a trial of medications aimed at weight loss unless BMI exceeds 50 (defined as superobese) in which case it is justifiable to proceed to surgery directly. The drugs which were available and licensed for weight loss were orlistat, sibutramine and rimonabant. Orlistat (a lipase inhibitor which prevents the absorption of around 30\% of dietary fat) is still available and can be purchased

Table 1 Weight related comorbidities

Cardiorespiratory
Hypertension
Obesity hypoventilation syndrome
Obstructive sleep apnoea
Asthma
Abdominal
Gastro-oesophageal reflux
Gallstones
Non-alcoholic fatty liver disease
Abdominal wall hernias
Metabolic
Diabetes mellitus
Dyslipidaemia
Hyperuricaemia
Androgenisation; polycystic ovary syndrome and infertility
Malignancy
Uterus
Colon
Ovary
Mechanical
Osteoarthritis of weight bearing joints
Abdominal wall herniae (eg, umbilical)
Urinary incontinence
Neurological disorders
Pseudotumour cerebri (intracranial hypertension)
Headache
Tremorive disorders
Intertrigo
Psoriasis of self-esteem

over the counter. Sibutramine (an appetite suppressant with $\beta 3$ agonist activity causing an increase in metabolic rate) was withdrawn due to cardiac risk, and rimonabant (a cannabinoid receptor antagonist aimed at reducing addictive behaviour) was withdrawn due to a link with depression leading to suicide. Table 2 lists the salient points in the NICE guidelines.

In the UK, around 700000 adults would be suitable for surgery under these guidelines and for NHS commissioning more stringent criteria than those defined by NICE have been introduced by many primary care trusts who now require a BMI of $>45$ with comorbidity or $>50$ alone to achieve funding.

There is no defined upper age limit, but the surgical risks are greater and the benefits less in older patients as cardiorespiratory complications of obesity progress and comorbidity, such as advanced joint problems and diabetic problems, become irreversible. The revised NICE guidelines removed the previous lower age limit of 18 years, substituting it with 'physiologically mature', and patients as young as 14 years have undergone bariatric surgery in this country. There is a huge capacity shortfall between patients who would be suitable and those that can be treated and a recurring utilitarian argument as to whether priority should be given to the very heavy patients with the greatest comorbidity who are no longer able to work and have the greater surgical risk, or the lower BMI patients with lower risk and shorter potential length of stay who are more likely to fully rehabilitate but are currently costing less in terms of comorbidity and indirect healthcare costs.

If surgery is indicated, it is necessary to assess a patient's medical and psychological suitability.

Table 2 National Institute for Health and Clinical Excellence (NICE) guidelines (CG46)

\footnotetext{
Consider surgery if $\mathrm{BMI}>40$, or $>35$ with significant comorbid conditions that could be improved by weight loss.

Surgery should only be undertaken by a multidisciplinary team that can provide:

Preoperative assessment and assessment of eating disorders Information on the various procedures, including weight loss and risks

Surgical and dietetic follow-up

Psychological support

Information on/access to plastic surgery

Access to and training in specialist equipment

Data collection and prospective audit

The patient has been receiving treatment in a specialised obesity clinic

Evidence that all appropriate and available non-surgical measures, including pharmacotherapy, have been tried but have failed to maintain weight loss if $\mathrm{BMI}<50$

No specific clinical or psychological contraindication to this type of surgery

Fit for anaesthesia and surgery

The patient commits to the need for long term follow-up
}

BMI, body mass index. 
Undiagnosed endocrine disorders such as hypothyroidism and adrenocortical disorders should be sought as a cause of obesity but they are very rarely found. ${ }^{10}$

Liver function tests should be performed but are frequently deranged from non-alcoholic fatty liver disease, a spectrum of liver disease extending from bland steatosis to cirrhosis from non-alcoholic steatohepatitis. ${ }^{11}$ Evidence of portal hypertension/liver failure, such as a depressed platelet count, raised bilirubin or deranged clotting, merits further investigation. One to two per cent of patients will have cirrhosis at the time of referral for surgery, the risk being highest in older diabetic males with a high waist-hip ratio-that is, android or central rather than gynaecoid or peripheral fat distribution. In the absence of liver failure, cirrhosis is not an absolute contraindication to bariatric surgery and although varices can lead to problems with dissection around the hiatus, the results of bariatric surgery in this patient group are acceptable. ${ }^{12}$

Many morbidly obese patients have independent cardiac risk factors such as diabetes, hypertension, dyslipidaemia and an increased risk of cardiovascular disease. ${ }^{13}$ For most patients, a resting ECG is adequate, with recourse to a stress echo or cardiopulmonary exercise testing in higher risk patients although this latter investigation to define the anaerobic threshold using a treadmill or exercise bike can be mechanically problematic in very large patients. The risk of obstructive sleep apnoea, as indicated by a high Epworth score, should be evaluated by overnight oximetry and patients with significant sleep apnoea should be stabilised on continuous positive airway pressure for at least a month prior to surgery and maintained on continuous positive airway pressure throughout their admission. ${ }^{14}$

A full dietetic history must be taken and the patient must be aware of the dietary restriction that will be placed on them and the need to take supplements with all of the malabsorptive procedures. ${ }^{15}$ The operations are designed to reduce the volumes ingested and induce satiety but the patient must be prepared to alter their dietary choices. Unlike volume eating, habits such as nibbling or grazing throughout the day on high fat energy dense foodstuffs such as crisps do not do well with surgery, and excess alcohol intake should be viewed as a relative contraindication to surgery.

Patients should be given access to the patent support network prior to surgery (eg, via www.bospa.org and www.wlsinfo.org.uk) and encouraged to attend support group meetings. Weight loss surgery is very good for weight loss and improving weight related comorbidity but does not address all the weight related problems, such as the cause of the overeating. A history of an abusive relationship or childhood abuse is common and while the relationship may be casual rather than causal, support must be available if the prop of comfort eating is to be permanently removed.
Patients must accept the importance of compliance with regular exercise for long term weight maintenance and should agree to aim to address this lifestyle change after surgery.

Assessment of patients should be entrusted to a multidisciplinary team with representation by, or access to, the following: specialist bariatric dieticians, bariatric surgeon, specialist anaesthetist/intensivist, bariatric specialist nurse, respiratory physician, endocrinologist, cardiologist, gastroenterologist, psychologist/psychiatrist and patient support group.

\section{Choice of procedure}

Operations designed to induce weight loss can be broadly divided into restrictive procedures, which reduce the intake of food, malabsorptive procedures, which interfere with the digestion and absorption of the ingested food, and combined restrictive/malabsorptive procedures, which do both. Bariatric procedures should be viewed as tools to assist with weight loss which work best when combined with lifestyle changes such as regular exercise, good dietary choices and sensible use of alcohol. Some potential patients are not suitable because they would not be prepared or able to make these changes and all procedures have a failure rate which is most often related to noncompliance.

\section{Malabsorptive procedures}

Malabsorptive operations are designed to induce weight loss by preventing ingested food from being adequately absorbed by the intestine. The earliest bariatric procedure was the jejuno-ileal bypass where most of the intestine is bypassed and the majority of food that is eaten is passed through undigested. Although highly effective, these types of operations can lead to severe malnutrition and life threatening metabolic complications, in some cases progressing to liver failure, and are no longer recommended as isolated procedures for obesity. Currently, all procedures are restrictive with or without an element of malabsorption.

\section{Gastric restrictive procedures}

This is a group of operations where weight loss is achieved by reducing the effective capacity of the stomach, thus restricting the intake of food to a point where this enforced strict diet ensures weight is lost. Because absorption of the ingested food by the intestine is normal, provided that a balanced diet is adhered to these operations do not cause nutritional failure, although patients are often advised to take vitamin and mineral supplements after surgery. With the exception of the endoscopically placed intragastric balloon, which is only for short term use and not a definitive procedure, these operations employ some form of stomach stapling or banding. The earliest purely restrictive procedure was the vertical banded gastroplasty but long term results were poor and this 
is rarely performed now. ${ }^{16}$ Currently, the most widely performed purely restrictive procedure is the laparoscopic adjustable gastric band. In this operation, using a keyhole or laparoscopic approach, the upper stomach is encircled with a doughnut-like inflatable balloon which is attached through a piece of latex tubing to an injection port which is placed under the skin. Injection of fluid into the port will inflate the balloon around the stomach to create a restriction to the intake of food, the capacity of the small pouch of stomach above the band determining the volume that can be eaten. ${ }^{17}$

\section{Combined restrictive and malabsorptive operations}

The most widely performed combined restrictive and malabsoptive bariatric procedure is the gastric bypass which can be performed through a single abdominal incision, or laparoscopically. ${ }^{18}{ }^{19}$ In this procedure, the stomach is completely closed off by a stapling device to create a small gastric pouch. The proximal small intestine is divided and the distal cut end of the jejunum anastomosed to the gastric pouch to create an alimentary limb. The proximal cut end of the jejunum is anastomosed to the side of the alimentary limb $75-150 \mathrm{~cm}$ beyond the gastroenterostomy as an entero-enterostomy. Food bypasses the distal stomach, duodenum and proximal jejunum and is separated from the pancreatic enzymes and bile until both reach the entero-enterostomy and enter the common channel, which should exceed $200 \mathrm{~cm}$. As a result, food absorption is impaired by shortening the length of the small intestine that is exposed to food, and delaying food mixing with pancreatic enzymes and bile. This assists in weight loss but the bowel will show adaptation and long term the effect of gastric bypass is predominantly due to gastric restriction. The technique of reconstruction employed in the gastric bypass creates two limbs of the small intestine in a Y shaped configuration. It is credited to the Swiss surgeon Cesar Roux and the procedure is often referred to as the Roux-en-Y gastric bypass.

An additional feature of the gastric bypass is that over ingestion of refined carbohydrate can lead to inappropriate excessive insulin release and a paradoxical fall in blood glucose. This hypoglycaemia creates unpleasant symptoms of nausea and lightheadedness ('dumping') and induces a degree of food aversion, particularly for refined carbohydrates, although this may not occur in type 2 diabetics with insulin resistance. Weight loss is generally greater than with a laparoscopic adjustable gastric band but there is a small risk of malnutrition and vitamin and mineral supplements are routinely given. The two other procedures performed are the sleeve gastrectomy/duodenal switch and the biliopancreatic diversion, which are more malabsorptive than gastric bypass. ${ }^{20}$ The sleeve gastrectomy entails removal of most of the body of stomach to leave a narrow tube of stomach, retaining the pylorus. The sleeve gastrectomy can be performed as a standalone procedure, working purely by gastric restriction, or be combined at the time or subsequently with a more extensive bypass of the intestine (duodenal switch) for additional weight loss. Unlike the gastric bypass, the pylorus is retained which prevents the dumping' effect. The biliopancreatic diversion involves a subtotal gastrectomy and an extensive bypass of the small intestine with a common channel of only 50 $\mathrm{cm}$. These latter procedures are generally reserved for patients with a BMI of over 60 and have a greater risk of diarrhoea and malnutrition but do give greater weight loss while allowing a more liberal intake of food in terms of volume. Eighty per cent of patients undergoing weight loss surgery in the UK will have either a gastric band or gastric bypass.

Giving a patient appropriate expectations and careful informed consent is crucial in this area. All bariatric surgical procedures carry a risk of complications and of treatment failure and although this is predominantly due to non-compliance, there has been a reclassification of bariatric surgery by one of the medical indemnity insurance companies who have this year moved it into a higher band than general surgery, with a $50 \%$ increase in premium as a reflection of the greater risk of litigation which it attracts.

\section{Results and data collection}

The impact of bariatric surgery on weight related comorbidity is dramatic, and superior to best medical therapy, ${ }^{21}$ with long term improvement or resolution of diabetes, hypertension, sleep apnoea, reflux and dyslipidaemia in over $70 \%$ of patients ${ }^{22-24}$ although long term data with the gastric band suggest that the impact on hypertension may not be maintained. ${ }^{25}$

In addition, following bariatric surgery, there is evidence for a reduction in cancer incidence, ${ }^{9}$ weight related comorbidity and all cause mortality, ${ }^{24}$ and, using Markov modelling, a prolongation in life expectancy. ${ }^{26}$ There is no defined upper age limit for bariatric surgery and although in the older patient one may not expect an impact on life expectancy, there is evidence that bariatric surgery in the over 60s can still lead to a significant positive impact on weight related comorbidity. ${ }^{27}$

Bariatric surgery has also been shown to improve quality of life (QOL), as measured by non-specific measures such as the Short Form 36 and the Nottingham Health Profile, and by obesity specific QOL tools, such as Obesity Specific QOL, Obesity Related Well Being Scale, Obesity Adjustment Survey-Short Form and BAROS (bariatric analysis and reporting outcome system) when compared with non-operated controls for all the commonly employed procedures. ${ }^{28-30}$ Bariatric surgery also provides improved body image perception. ${ }^{30}$ These changes are evident within the first few months after surgery and maintained long term. ${ }^{29}{ }^{30} \mathrm{~A}$ 
bariatric surgery database has now been established for UK patients (http://nbsr.e-dendrite.com). Data entry is a requirement for compliance with most primary care trust held contracts, and although optional for private patients it is felt that the majority of cases are being entered.

The database collects demographic data, data on comorbid conditions, details of operative procedure and postoperative complications, and follow-up data in terms of weight loss, early readmission and impact on comorbidity.

The UK database is still evolving but there are already more than 12000 patients registered on it. A current shortfall is a lack of quantification when describing comorbidity such that a patient who had experienced poor blood pressure control on three agents prior to surgery, who becomes well controlled on a single agent, will still be recorded as 'hypertension on treatment', and improved diabetic control despite a drastic reduction in insulin dose will still be recorded as 'diabetic on insulin'. Data capture on issues such as pregnancy outcomes would be welcomed as increasing numbers of young women are having surgery, ${ }^{31}$ and date on return to employment would be of value in assessing the impact on indirect healthcare costs, but the database is still in its early stages and responding to feedback from its users.

Treatment failures can occur with bariatric surgery and setting realistic expectations at the outset is essential. In one of the very few prospective randomised studies comparing gastric banding and gastric bypass, excess weight loss at 4 years was $68 \% \pm 19 \%$ for the gastric bypass compared with $45 \% \pm 28 \%$ for the gastric band. Treatment failure, defined as loss of less than $20 \%$ of excess weight, was observed in $16.7 \%$ of gastric band patients compared with $0 \%$ of gastric bypass patients. ${ }^{32}$

To some extent this branch of surgery is becoming a victim of its own success, and there is a huge shortfall in our capacity to perform this surgery given the number of potential patients and the increasing incidence of obesity. Currently more than 10 times as many patients die from obesity than have surgery for obesity each year and while for the individual patient bariatric surgery can have a dramatic impact on their quality and quantity of life, this shortfall must be addressed if bariatric surgery is to have any impact on population statistics. $^{33}$

\section{Competing interests None.}

Provenance and peer review Commissioned; externally peer reviewed.

\section{References}

1. Peeters A, Barendregt JJ, Willekens F, et al. Obesity in adulthood and its consequences for life expectancy: a life-table analysis. Ann Intern Med 2003;138:24-32.

2. Department of Health. Summary of Intelligence on Obesity. London: Department of Health, 2004.
3. Physical status: the use and interpretation of anthropometry. Report of WHO expert committee. World Health Organ Tech Rep Ser 1995;854:1-452.

4. Huxley R, Mendis S, Zheleznyakov E, et al. Body mass index, waist circumference and waist:hip ratio as predictors of cardiovascular risk-a review of the literature? Eur J Clin Nutr 2010;64:16-22.

5. Qiao Q, Nyamdorj R. Is the association of type II diabetes with waist circumference or waist-to-hip ratio stronger than that with body mass index? Eur J Clin Nutr 2010;64:30-4.

6. Price GM, Uauy R, Breeze E, et al. Weight, shape, and mortality risk in older persons: elevated waist-hip ratio, not high body mass index, is associated with a greater risk of death. Am J Clin Nutr 2006;84:449-60.

7. Lubliner C. Are we two dimentional? 2 December 2007 http:// cobylubliner.wordpress.com (accessed 24 January 2011).

8. National Institute for Health and Clinical Excellence. Obesity-guidance on the prevention, identification, assessment and management of overweight and obesity in adults and children. London: The Stationery Office, 2006. http://www.nice.org (accessed 24 January 2011).

9. Gummesson A, Sjostrom L, Lystig T, et al. Effects of bariatric surgery on cancer incidence in Swedish obese subjects. Int J Obes 2008;32:S24.

10. Wilding PH. Pathophysiology and aetiology of obesity. Medicine 2006:34;501-5.

11. Neuschwander-Terti BA. Fatty liver disease and the metabolic syndrome. Curr Opin Gastroenterol 2007:23;193-8.

12. Dallal RM, Mattar SG, Lord JL, et al. Results of laparoscopic gastric bypass in patients with cirrhosis. Obes Surg 2004;14:47-53.

13. Hubert HB, Feinleib M, McNamara PM, et al. Obesity as an independent risk factor for cardiovascular disease: a 26-year follow-up of participants in the Framingham Heart Study. Circulation 1983;67:968-77.

14. Young T, Peppard PE, Taheri S. Excess weight and sleepdisordered breathing. J Appl Physiol 2005;99:1592-9.

15. Bloomberg RD, Fleishman A, Nalle JE, et al. Nutritional deficiencies following bariatric surgery: what have we learned? Obes Surg 2005;15:145-54.

16. Mason EE. Vertical banded gastroplasty for obesity. Arch Surg 1982;117:701-6.

17. Ren CJ, Fielding GA. Laparoscopic adjustable gastric banding: surgical technique. J Laparoendosc Adv Surg Tech A 2003;13:257-63.

18. Sugerman HJ, Kellum JM, Engle KM, et al. Gastric bypass for treating severe obesity. Am J Clin Nutr 1992;55(2 Suppl):560S-6S.

19. Oliak D, Ballantyne GH, Weber P, et al. Laparoscopic Rouxen-Y gastric bypass: defining the learning curve. Surg Endosc 2003;17:405-8.

20. Scopinaro N, Adami GF, Marinari GM, et al. Biliopancreatic diversion. World J Surg 1998;22:936-46.

21. Dixon JB, O'Brien PE, Playfair J, et al. Adjustable gastric banding and conventional therapy for type 2 diabetes: a randomized controlled trial. JAMA 2008;299:316-23.

22. Puzziferri N, Austrheim-Smith IT, Wolfe BM, et al. Three-year follow-up of a prospective randomized trial comparing laparoscopic versus open gastric bypass. Ann Surg 2006;243:181-8.

23. Sjöström L, Lindroos AK, Peltonen M, et al. Lifestyle, diabetes, and cardiovascular risk factors 10 years after bariatric surgery. N Engl J Med 2004;351:2683-93. 
24. Sjöström L, Narbro K, Sjöström CD, et al. Effects of bariatric surgery on mortality in Swedish obese subjects. N Engl J Med 2007;357:741-52.

25. Karlsson J, Taft C, Rydén A, et al. Ten-year trends in healthrelated quality of life after surgical and conventional treatment for severe obesity: the SOS intervention study. Int J Obes (Lond) 2007;31:1248-61.

26. Schauer DP, Arterburn DE, Livingston EH, et al. Decision modeling to estimate the impact of gastric bypass surgery on life expectancy for the treatment of morbid obesity. Arch Surg 2010;145:57-62.

27. Sugerman HJ, DeMaria EJ, Kellum JM, et al. Effects of bariatric surgery in older patients. Ann Surg 2004;240:243-7.

28. Livingston EH, Fink AS. Quality of life: cost and future of bariatric surgery. Arch Surg 2003;138:383-8.

29. Hell E, Miller KA, Moorehead MK, et al. Evaluation of health status and quality of life after bariatric surgery: comparison of standard Roux-en-Y gastric bypass, vertical banded gastroplasty and laparoscopic adjustable silicone gastric banding. Obes Surg 2000;10:214-19.

30. Sarwer DB, Wadden TA, Moore RH, et al. Changes in quality of life and body image after gastric bypass surgery. Surg Obes Rel Dis 2010:6;608-14.

31. Sheiner E, Balaban E, Dreiher J, et al. Pregnancy outcome in patients following different types of bariatric surgeries. Obes Surg 2009;19:1286-92.

32. Nguyen NT, Slone JA, Nguyen XM, et al. A prospective randomized trial of laparoscopic gastric bypass versus laparoscopic adjustable gastric banding for the treatment of morbid obesity: outcomes, quality of life, and costs. Ann Surg 2009:250;631-41.

33. Picot J, Jones J, Colquitt JL, et al. The clinical effectiveness and cost-effectiveness of bariatric (weight loss) surgery for obesity: a systematic review and economic evaluation. Health Technol Assess 2009;13:1-190, 215-357. 